\title{
Growth references for Japanese individuals with Noonan syndrome
}

\author{
Tsuyoshi Isojima', Satoru Sakazume ${ }^{2}$, Tomonobu Hasegawa ${ }^{3}$, Tsutomu Ogata ${ }^{4}$, Toshio Nakanishi ${ }^{5}$, \\ Toshiro Nagai ${ }^{2}$ and Susumu Yokoya ${ }^{6}$
}

BACKGROUND: Noonan syndrome (NS) is a clinically and genetically heterogeneous syndrome characterized by distinctive facial features, short stature, congenital heart diseases, and other comorbidities. NS-specific growth charts are essential for NS care, but currently no such charts are available for Asian populations.

METHODS: We conducted a nationwide survey by collaborating with three academic societies in Japan. We obtained the data of 356 clinically diagnosed NS subjects from 20 hospitals. The Lambda-Mu-Sigma method was used for establishing growth charts.

RESULTS: A total of 308 subjects (males: 159 and females: 149) were analyzed after excluding 48 subjects because of missing auxological data (26 subjects), presence of complications affecting growth (5 subjects), and extreme longitudinal growth aberrations which lay more than three standard deviation scores from the mean in this population (17 subjects). Genetic analyses were performed in 150 patients (48.7\%); 103 (68.7\%) were reported to have some abnormalities in the known causative genes. Cardiovascular diseases were found in 256 patients (83.1\%). The NS-specific height, weight, and BMI charts were constructed with 3,249 mixed longitudinal and cross-sectional measurements.

CONCLUSION: Growth standards for Japanese individuals with NS were established. These charts are expected to be used in various clinical settings.

N oonan syndrome (NS) is a clinically and genetically heterogeneous syndrome characterized by short stature, chest deformity, congenital heart disease, distinctive facial features that change with age, and other comorbidities $(1,2)$. The clinical features in NS can vary within the same family but become more subtle in adult life (3). NS is relatively common, with an estimated prevalence of between 1 in 1,000 to 1 in 2,500 live births $(2,4,5)$. Based on the cardinal features, the follow-up and comprehensive care for NS patients require a multidisciplinary team of specialists. As NS is characterized by marked variable dysmorphic appearances (6), it is often diagnosed by pediatric geneticists. Pediatric cardiologists also have an important role in NS care as it is the second most common syndromic cause of congenital heart disease, exceeded in prevalence only by trisomy 21 (7). In addition, children with NS often present to pediatric endocrinologists because of their short stature, delayed puberty, or undescended testes in males (3).

The protein-tyrosine phosphatase, nonreceptor type 11 (PTPN11) gene was the first causative gene identified in NS patients. It encodes a tyrosine phosphatase protein involved in the Ras/mitogen-activated protein kinase signaling pathway (8). The protein is expressed throughout the body and plays a crucial role in cellular response to growth factors, hormones, cytokines, and cell adhesion molecules. There is increasing evidence that germline mutations in the genes involved in the Ras/mitogen-activated protein kinase signaling pathway cause NS and Noonan-related syndrome (9). While mutations in such genes were identified in $\sim 60-70 \%$ of patients with NS phenotype $(10,11)$, the diagnosis of NS remains clinical $(2,12)$. Several scoring systems are currently available to aid in the diagnostic process, with the one developed by van der Burgt et al. (13) in 1994 being the most widely used.

At present, there are disease-specific growth charts for several syndromes, such as Prader-Willi syndrome, Down syndrome, Williams syndrome, and Turner syndrome (14-18). These charts are generally used in the clinical setting for monitoring the growth and development of patients with these syndromes and allow the identification of those with severe growth deficit who may need additional medications. To date, three NS-specific growth curves have been established in Brazil, North America, and Europe. Two of them were developed with data mainly collected by pediatric geneticists $(19,20)$, while the other was constructed with data gathered by pediatric endocrinologists and cardiologists (21). Evidently, NS-specific charts should be created with data collected by a multidisciplinary team of specialists who is involved in the follow-up of NS patients. To that end, we conducted a nationwide survey of pediatric geneticists, pediatric cardiologists, and pediatric

\footnotetext{
'Department of Pediatrics, Graduate School of Medicine, The University of Tokyo, Tokyo, Japan; ${ }^{2}$ Department of Pediatrics, Dokkyo Medical University Koshigaya Hospital, Koshigaya, Japan; ${ }^{3}$ Department of Pediatrics, Keio University School of Medicine, Tokyo, Japan; ${ }^{4}$ Department of Pediatrics, Hamamatsu University School of Medicine, Hamamatsu, Japan; ${ }^{5}$ Department of Pediatric Cardiology, The Heart Institute, Tokyo Women's Medical University, Tokyo, Japan; ${ }^{6}$ Department of Medical Subspecialties, National Center for Child Health and Development, Tokyo, Japan. Correspondence: Susumu Yokoya (yokoya-s@ncchd.go.jp)

Received 1 June 2015; accepted 17 September 2015; advance online publication 6 January 2016. doi:10.1038/pr.2015.254
} 
endocrinologists who are responsible for Japanese NS patients to establish growth standards for Japanese patients with NS.

\section{RESULTS}

Twenty of the 31 hospitals (64.5\%) responded to our questionnaire, and the data of 356 subjects clinically diagnosed with NS were obtained. From these data, a total of 48 subjects were excluded because of missing auxological data (26 subjects), presence of complications that affected growth (5 subjects), and extreme longitudinal growth aberrations which lay more than three standard deviation scores (SDS) from the mean in this population (17 subjects). The remaining 308 subjects (159 males and 149 females) were analyzed. Their birth years ranged from 1970 to 2012 (median: 1998). Gestational age was $38.4 \pm 2.4 \mathrm{wk}(n=247)$, birth length $48.2 \pm 3.0 \mathrm{~cm}$ (male, $n=94)$ and $47.8 \pm 3.4 \mathrm{~cm}$ (female, $n=104$ ), birth weight $3.04 \pm 0.55 \mathrm{~kg}$ (male, $n=137$ ) and $3.03 \pm 0.57 \mathrm{~kg}$ (female, $n=141$ ), target height $170.9 \pm 5.3 \mathrm{~cm}$ (male, $n=58$ ) and $157.5 \pm 4.8 \mathrm{~cm}$ (female, $n=66$ ). Target height was calculated by the formula adjusted for Japanese before the secular trend reached a plateau (22). Table 1 shows the cumulative cardiac findings of the 308 patients. Cardiovascular disease was seen in 256 patients $(83.1 \%)$. Genetic analyses were performed in 150 patients (48.7\%), among them 103 individuals (68.7\%) were found to have abnormalities in the causative genes (i.e., PTPN11: 67, KRAS: 3, SOS1: 7, RAF1: 14, BRAF: 7, SHOC2: 3, and RIT1: 2).

To establish the growth standards, 3,249 height measurements (males: 1,674 and females: 1,575) and 3,111 weight measurements (males: 1,564 and females: 1,547) were eventually included after the exclusion of 23 height measurements and 19 weight measurements derived from three outliers. Scatter plots

Table 1. Cardiac findings in our series of 308 patients with NS

\begin{tabular}{lcc}
\hline Cardiac defect & Number $^{\mathrm{a}}$ & Rate (\%) \\
\hline Pulmonary stenosis & 162 & 52.6 \\
Hypertrophic cardiomyopathy & 84 & 27.3 \\
Atrial septal defect (ostium secundum type) & 66 & 21.4 \\
Ventricular septal defect & 35 & 11.4 \\
Mitral valve anomalies & 19 & 6.2 \\
Patent ductus arteriosus & 10 & 3.2 \\
Aortic valve stenosis & 9 & 2.9 \\
Atrioventricular septal defect & 3 & 0.97 \\
Aortic coarctation & 2 & 0.65 \\
Pulmonary arterial hypertension & 2 & 0.65 \\
Tetralogy of Fallot & 2 & 0.65 \\
Bicupsid aortic valve & 1 & 0.32 \\
Coronary artery abnormality & 1 & 0.32 \\
Double outlet right ventricle & 1 & 0.32 \\
Aortic valve regurgitation & 1 & 0.32 \\
Truncus arteriosus communis & 1 & 0.32 \\
No finding & 52 & 16.9 \\
\hline NS,Noon syndrom & & \\
\hline
\end{tabular}

NS, Noonan syndrome.

aNumber indicates the cumulative total number of patients. of height and weight measurements were plotted on growth charts for normal population in Supplementary Figures S1 and S2 online, respectively. The majority of subjects (males: $n=126(79.2 \%)$ and females: $n=127(85.2 \%))$ had repeated measurements (male: median 10, range 2-63; female: median 11, range 2-46). Table 2 lists the number of measurements by sex and age. There was no statistically significant difference in height SDS between those with and without genetic analysis (difference: $0.076,95 \%$ confidence interval (CI): -0.020 to $0.17, P=0.12$ ). In contrast, height SDS in patients with PTPN11 mutation was significantly smaller than that with other gene mutation (difference: $0.39,95 \%$ CI: $0.26-0.53, P<0.0001$ ).

Centile curves were fitted to the data using the LambdaMu-Sigma (LMS) method. For height, the distribution was generally assumed normal, while for weight and BMI, there was appreciable skewness, and the age-varying power transformations were adjusted. Equivalent degrees of freedom for (L, M, S) of height, weight, and BMI for males are (0, $12,10)$ with age rescaled, $(4,9,5)$ with age rescaled, and $(3,7,4)$ with age transformed, respectively. Equivalent degrees of freedom for (L, M, S) of height, weight, and BMI for females are $(0,10,7)$ with age rescaled, $(4,11,5)$ with age rescaled, and $(3,6,4)$ with age transformed, respectively. Supplementary Tables S1-S3 online provide values for $\mathrm{L}, \mathrm{M}$, and $\mathrm{S}$ of height, weight, and BMI by sex and age,

Table 2. Age distribution of numbers of measurements used for analysis

\begin{tabular}{|c|c|c|c|c|c|c|}
\hline \multirow{2}{*}{$\begin{array}{l}\text { Age } \\
\text { (years) }\end{array}$} & \multicolumn{2}{|c|}{ Height } & \multicolumn{2}{|c|}{ Weight } & \multicolumn{2}{|c|}{ Body mass index } \\
\hline & Boy & Girl & Boy & Girl & Boy & Girl \\
\hline 0 & 300 & 279 & 322 & 311 & 300 & 279 \\
\hline 1 & 175 & 174 & 164 & 177 & 164 & 174 \\
\hline 2 & 128 & 121 & 125 & 121 & 125 & 121 \\
\hline 3 & 155 & 125 & 144 & 127 & 144 & 125 \\
\hline 4 & 132 & 94 & 126 & 91 & 126 & 91 \\
\hline 5 & 128 & 75 & 121 & 77 & 121 & 75 \\
\hline 6 & 105 & 90 & 99 & 86 & 99 & 86 \\
\hline 7 & 79 & 78 & 72 & 78 & 72 & 78 \\
\hline 8 & 70 & 87 & 69 & 87 & 69 & 87 \\
\hline 9 & 52 & 63 & 51 & 61 & 51 & 61 \\
\hline 10 & 46 & 60 & 46 & 57 & 46 & 57 \\
\hline 11 & 58 & 48 & 51 & 44 & 51 & 44 \\
\hline 12 & 47 & 47 & 41 & 45 & 41 & 45 \\
\hline 13 & 43 & 47 & 33 & 45 & 33 & 45 \\
\hline 14 & 43 & 44 & 33 & 42 & 33 & 42 \\
\hline 15 & 35 & 30 & 25 & 27 & 25 & 27 \\
\hline 16 & 21 & 30 & 14 & 27 & 14 & 27 \\
\hline 17 & 16 & 24 & 10 & 22 & 10 & 22 \\
\hline 18 & 9 & 13 & 4 & 9 & 4 & 9 \\
\hline 19 & 10 & 13 & 5 & 5 & 5 & 5 \\
\hline $20+$ & 22 & 33 & 9 & 8 & 9 & 8 \\
\hline Total & 1,674 & 1,575 & 1,564 & 1,547 & 1,542 & 1,508 \\
\hline
\end{tabular}



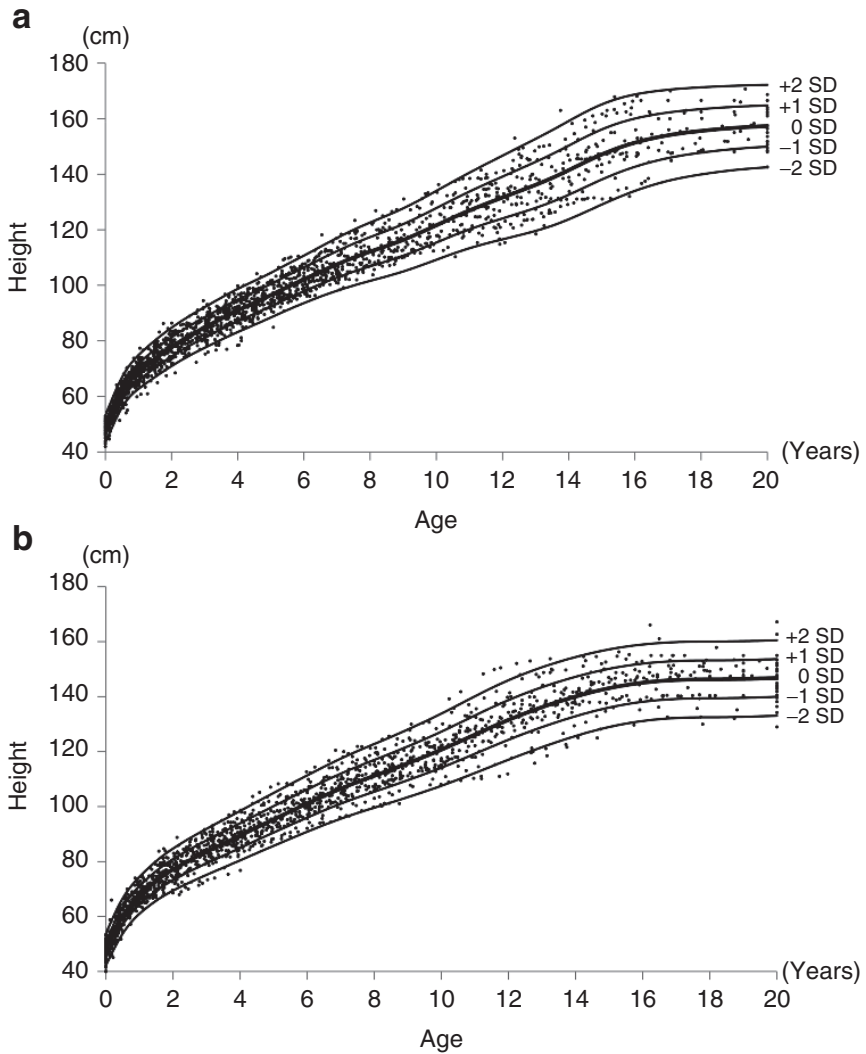

Figure 1. Scatter plots and smoothing curves of $+2 S D,+1 S D$, median, -1 SD, and -2 SD of height in Japanese (a) male and (b) female subjects with NS.

respectively. Clinical growth charts with scatter plots of subjects for height, weight, and BMI are shown in Figures 1-3, respectively. A comparison with previously published mean height data for NS (19-21) was given in Figure 4. Growth curves for height, weight, and BMI are superimposed on those of normal population as shown in Supplementary Figures S3-S5 online, respectively. Male weight and BMI charts are superimposed on those of female as shown in Supplementary Figure S6 online.

\section{DISCUSSION}

From the nationwide survey data of NS patients followed up by specialists from different fields, we have established growth references for Japanese NS patients. To our knowledge, these are the first NS-specific growth references in an Asian population. They were constructed by the LMS method, which we believe to be one of the most widely applied approaches (23). The LMS method is applicable to not only cross-sectional data but also longitudinal data, if all subjects were measured broadly and the number of measurements per individual did not reflect the growth charts (24). In this study, we collected the data in the following way so that certain individuals would not have much influence on the established charts. Our questionnaire required the minimal interval between two anthropometric values to be 1 mo when the subjects were measured before $1 \mathrm{y}$ of age or $3 \mathrm{mo}$ after $1 \mathrm{y}$ of age. In fact, after $10 \mathrm{y}$
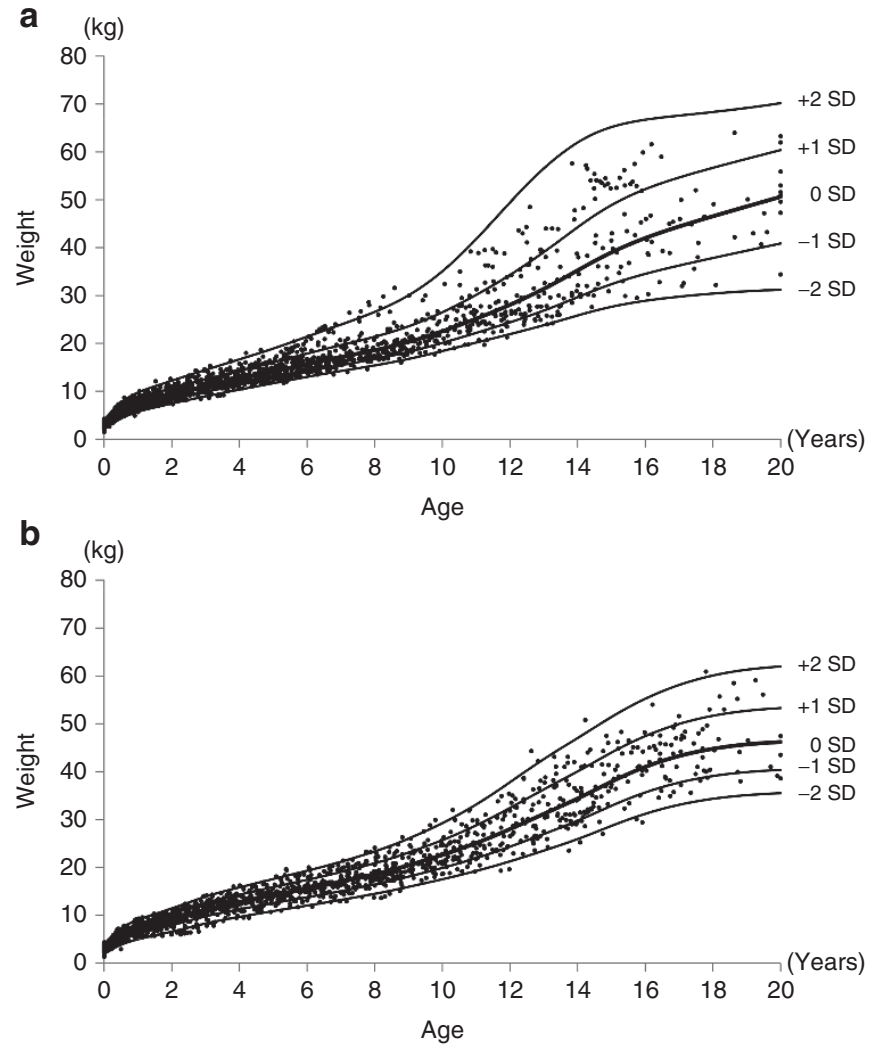

Figure 2. Scatter plots and smoothing curves of $+2 \mathrm{SD},+1 \mathrm{SD}$, median, -1 SD, and -2 SD of weight in Japanese (a) male and (b) female subjects with NS. NS, Noonan syndrome.

of age, the intervals between two anthropometric values were more than half a year.

The number of subjects analyzed in this study $(n=308)$ was sufficient compared with the numbers analyzed in the construction of the other three existing NS-specific charts ( $n=112,137$, and 144, respectively) (19-21). The diagnosis of NS in the subjects in this study was very reliable because it was performed by specialists certified for NS care in Japan by one of the three collaborating societies. The diagnosis was further validated using the scoring system developed by van der Burgt et al. (13). All auxological data were properly selected by excluding data with previous growth-promoting treatment, reported complications that may affect growth, and extreme longitudinal growth aberration. In addition, the percentage of detected abnormalities in the causative genes $(68.7 \%)$ was reasonable compared to the reported ratios $(10,11)$. Notably, the frequency of cardiovascular disease in this cohort $(83.1 \%)$ is very similar to that of a recent report of 293 NS patients (81\%) (25). Taken together, the newly established charts can be considered as representative of the growth in individuals with NS who are ordinarily seen in clinical practice.

The growth pattern of height was in line with previous reports $(19-21,26)$. The average birth length is slightly shorter but within normal limit; however, there is a rapid decline in length/ height SDS for normal population during the first year of life. Thereafter, mean height lies below the -2 SDS line for normal 

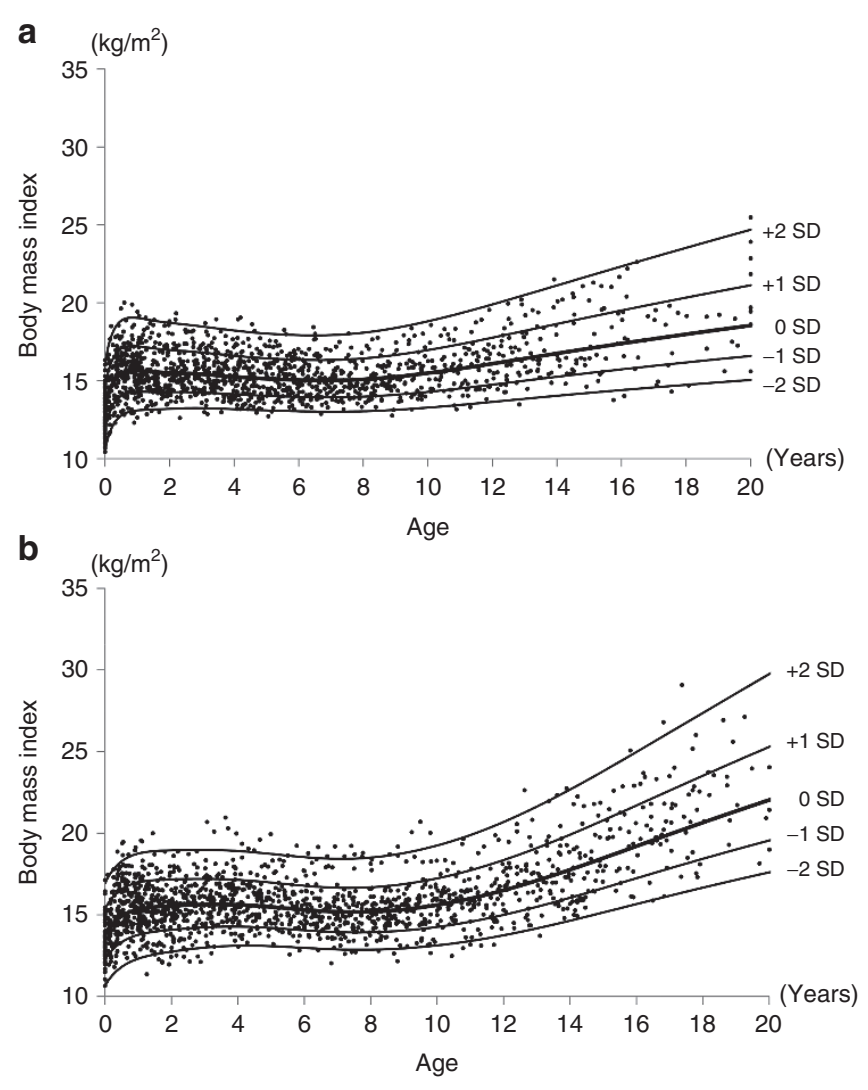

Figure 3. Scatter plots and smoothing curves of $+2 S D,+1 S D$, median, -1 SD, and -2 SD of body mass index in Japanese (a) male and (b) female subjects with NS. NS, Noonan syndrome.

population until $12 \mathrm{y}$ in boys and $10 \mathrm{y}$ in girls. Afterwards, there is a further decline due to delayed pubertal spurt, and then, the height SDS for normal population tends to recover. The adult height from the newly established growth charts is $157.3 \pm 7.4 \mathrm{~cm}$ for boys $(-2.3 \pm 1.3 \mathrm{SDS})$ and $146.8 \pm 6.9 \mathrm{~cm}$ for girls $(-2.1 \pm 1.3 \mathrm{SDS})$, respectively, when it is defined as the mean height at the age of $20 \mathrm{y}$. These adult height SDS for normal population are considered to be valid when compared to adult height SDS reported in other countries (male: -2.5 to -1.4 , female: -2.2 to -1.8$)(19-21,26,27)$, although small differences likely due to the different definitions of adult height were noted. In view of these, our NS-specific growth standards give adequate information about the natural growth of NS and are expected to be useful for evaluating height in various clinical settings such as potential growth-promoting treatments in NS.

With regard to the growth pattern of weight, birth weight is within normal limit; however, there is also a rapid decline in weight SDS to approximately -2 SDS for normal population during the first year of life, which may reflect the feeding problem in NS. Thereafter, growth retardation of weight is not apparently progressive. There seems to be a sexual difference of growth pattern of weight in older ages. Distributions of weight in male NS individuals are wider than those in female NS individuals, although it is difficult to interpret the implication with only auxological measurements in this study. On the other hand, BMI is similar to that of normal populations until $\sim 5$ y of age, but gradual increase after BMI rebound age is milder than that of normal population. These results are in line with the Brazilian BMI charts (20) and the reportedly low prevalence of overweight in adult NS patients (28). In addition, it is noteworthy that there is also a sexual difference in the growth pattern of BMI; gradual increase after BMI rebound age in male is milder than that in female. The interpretation of this difference remains unclear in the present study.

A difference in height SDS was detected throughout the whole period between those with PTPN11 mutation and with other gene mutation (difference: 0.39, 95\% CI: 0.26-0.53, $P<0.0001$ ). Although it may be due to the fact that patients with PTPN11 mutation often have low concentrations of insulin-like growth factor 1 (7), there are conflicting data on the severity of short stature between PTPN11 mutation-positive and mutation-negative groups. In some studies, there were no differences in growth parameters between 51 PTPN11 mutation-positive and 64 mutation-negative subjects (29), as was the case in an analysis of adult height in 56 patients (30). In another study, $82 \%$ of 34 PTPN11 mutation-positive cases and $57 \%$ of the 23 PTPN11 mutation-negative subjects had a height of less than -2 SDS (mean -3.1 SDS and -2.4 SDS, respectively) (31). There are similar reports that PTPN11 mutation-positive subjects are shorter than PTPN11 mutation-negative subjects $(32,33)$. Although further investigation is needed, our study provides additional clinical data that NS individuals with PTPN11 mutation might be 0.39 SDS shorter than those with other gene mutations.

There are several limitations in this study. First, the number of measurements of near-adult ages, especially in weight and BMI (Table 2), is low. Therefore, our charts may not be sufficiently reliable at these ages. Notably, this limitation is shared by all the other NS-specific charts (19-21). Nonetheless, growth patterns shown by our charts are in line with other studies of longitudinal natural history of NS $(27,30)$. The adult heights from our charts were also valid when compared to other reports discussed above. The second limitation is a selection bias. For establishing disease-specific charts, recruiting unbiased subjects is challenging but nevertheless crucial. As NS is a clinically and genetically heterogeneous syndrome, it is critical to determine which subjects should be analyzed. For example, if we collected only the data of NS patients being confirmed genetically, there would have been an inherent selection bias because individuals with clinically confirmed NS may not necessarily undergo genetic analyses nor receive genetically confirmed diagnosis. However, the difference in height SDS between those with and without genetic analyses was not statistically significant, which suggested that these two groups had similar characteristics in height. Another type of selection bias regarding unreliable clinical diagnosis may derive from clinicians' limited specialty. However, this was largely overcome because this study was a nationwide survey of clinically diagnosed NS patients cared for by specialists for NS care.

In conclusion, we constructed the growth standards for Japanese individuals with NS using 3,249 measurements 
from 308 NS patients nationwide. These are the first Asian NS-specific growth charts and are expected to be widely used in various clinical settings.

\section{METHODS}

\section{Subjects}

The nationwide survey of NS was conducted in collaboration with three academic societies in Japan (i.e., The Japan Society of Pediatric Genetics; The Japanese Society of Pediatric Cardiology and Cardiac Surgery; and The Japanese Society for Pediatric Endocrinology). We collected the data of patients in 31 hospitals nationwide who had been clinically diagnosed with NS by specialists certified by one of the three societies, and whose birth year was not earlier than 1970 considering the secular trend in height in Japan (17). We sent a questionnaire that included auxological measurements together with a minimal set of clinical and genetic data. Data on height and weight were collected in a mixed longitudinal and cross-sectional fashion, and the minimal interval between two anthropometric values was 1 mo when the subjects were measured before $1 \mathrm{y}$ of age or 3 mo after $1 \mathrm{y}$ of age. BMI was calculated as weight in kilograms divided by the square of height in meters. Clinical and genetic data included the following: clinical features according to the scoring system developed by van der Burgt et al. (13), classification of congenital heart disease, sex, birth year, birth weight, birth length, gestational periods, complications, comorbidities such as growth hormone deficiency, age at start of puberty, parents' height, family history, initial clues to the diagnosis, results of genetic analysis if available, and identified mutation(s) in the causative genes. We excluded subjects with missing description of auxological data, reported complications that significantly affected their growth, and extreme longitudinal growth aberrations which lay more than three SDS from the mean in this population. Subjects with growthpromoting treatment were included only if they had pretreatment auxological data, but measurements after the commencement of the growth-promoting therapy were excluded from our analysis. This study was performed with approval from National Center for Child Health and Development Ethics Committee and all the other Ethics Committees in institutes which participated in this study. We did not collect any information which may identify individuals, such as name and address, and so did not obtain informed consent from participants. The Japanese guidelines permit the use of data from epidemiological survey without consent if the data are anonymous. The information on the present survey has been disclosed to the public on the website of National Center for Child Health and Development Ethics Committee at http://www.ncchd. go.jp/center/information/epidemiology/index.html.

\section{Methods}

Data cleaning was performed at several stages. Bivariate plots of height and weight were used to identify gross disproportion. Data points were scrutinized, going back to the source data if necessary, and descriptive errors were corrected. If a value was deemed highly unlikely (more than 3 SDS from the mean in the population), even though there was no evidence of a descriptive error, such a point was deleted.

To establish the growth charts, the LMS method (34) was used, with the assumption that the data can be transformed to normality by a suitable power transformation $(\mathrm{L})$, and the distribution summarized by the median $(\mathrm{M})$ and coefficient of variation $(\mathrm{S})$. Using penalized likelihood, three curves (L, M, and S) can be fitted as cubic splines by nonlinear regression, and the extent of smoothing was controlled by equivalent degrees of freedom. The values of $\mathrm{L}, \mathrm{M}$, and S were constrained to change smoothly with age, and fitted values can be used to construct any required centile curves. Fitting and smoothing were done with lmsChartMaker Pro ver.2.3 (Medical Research Council, London, UK). The SD score (Z-score) of each measurement (y value) could be calculated from the $\mathrm{L}, \mathrm{M}$, and $S$ curves, using values appropriate for the age and gender, by the following equation: $Z=\left[(y / M)^{\mathrm{L}}-1\right]$ / $(\mathrm{L} \times \mathrm{S})$, and if $\mathrm{L}=0, \mathrm{Z}=\ln (\mathrm{y} / \mathrm{M}) / \mathrm{S}$.

\section{Statistical Analysis}

The results are expressed as the mean \pm SD or by frequency and percentage. To investigate the height difference between those with
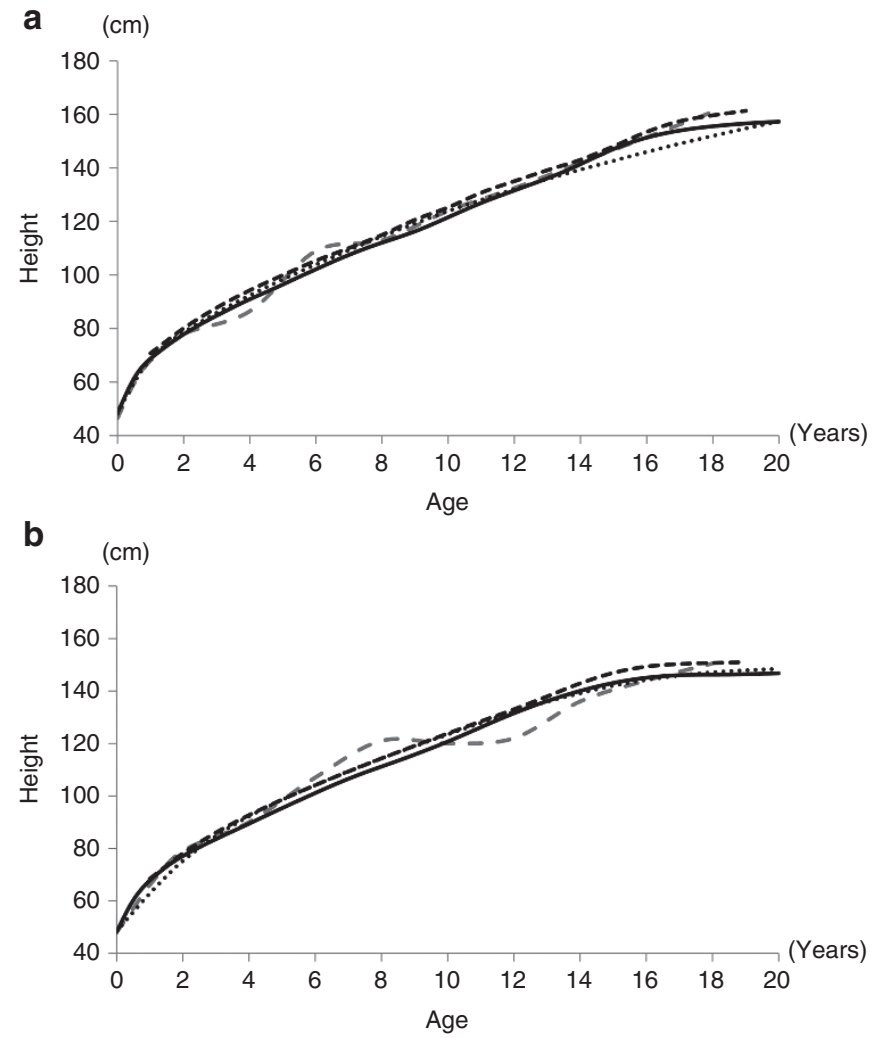

Figure 4. Comparison of Japanese NS mean height curves with previously published foreign curves, (a) male and (b) female, respectively. Black, black dotted, black dashed, and gray broken lines indicate Japanese, Brazilian, European, and North American NS mean height curves, respectively. NS, Noonan syndrome.

and without genetic analyses, height SDS, which were calculated by Japanese standards for normal children by sex and age (35), were compared with ANOVA considering the adjustment for the number of measurements in each subjects. Similar analyses were performed with other factors. These analyses were performed by JMP 8 (SAS Institute, Cary, NC); $P$ values $<0.05$ were considered statistically significant.

\section{SUPPLEMENTARY MATERIAL}

Supplementary material is linked to the online version of the paper at http://www.nature.com/pr

\section{ACKNOWLEDGMENTS}

We thank the following members of this nationwide survey study: Hironori Tanahashi, Department of Pediatrics, Asahikawa Medical College School of Medicine; Masato Yokozawa, Department of Pediatrics, Hokkaido Medical Center for Child Health and Rehabilitation; Yoko Aoki, Department of Medical Genetics, Tohoku University School of Medicine; Ikuma Fujiwara, Department of Pediatrics, Tohoku University School of Medicine; Hitoshi Horigome, Department of Child Health, Graduate School of Comprehensive Human Science, University of Tsukuba; Hirofumi Ohashi, Division of Medical Genetics, Saitama Children's Medical Center; Hiroshi Mochizuki, Division of Endocrinology and Metabolism, Saitama Children's Medical Center; Chie Takahashi, Yasuhiro Naiki, Reiko Horikawa, Division of Endocrinology and Metabolism, National Center for Child Health and Development; Reina Ishizaki, Hiroyuki Yamagishi, Department of Pediatrics, Keio University School of Medicine; Kenjiro Kosaki, Center for Medical Genetics, Keio University School of Medicine; Hiroshi Yoshihashi, Division of Medical Genetics, Tokyo Metropolitan Children's Medical Center; Yukihiro Hasegawa, Division of Endocrinology and Metabolism, Tokyo Metropolitan Children's Medical Center; In-Sam Park, Department of Pediatrics, Sakakihara Heart Institute; Sachiko Kitanaka, Department of Pediatrics, Graduate School of Medicine, The University of Tokyo; Kenji Kurosawa, Division of 
Medical Genetics, Kanagawa Children's Medical Center, Masanori Adachi, Department of Endocrinology and Metabolism, Kanagawa Children's Medical Center; Ayumi Uematsu, Department of Endocrinology and Metabolism, Shizuoka Children's Hospital; Yasuo Ono, Department of Cardiology, Shizuoka Children's Hospital; Haruo Mizuno, Shinji Saito, Department of Pediatrics and Neonatology, Nagoya City University Graduate School of Medical Sciences; Masako Izawa, Takashi Hamajima, Division of Endocrinology and Metabolism, Aichi Children's Health and Medical Center; Yasuko Shoji, Shinobu Ida, Department of Pediatric Gastroenterology, Nutrition, and Endocrinology, Osaka Medical Center and Research Institute for Maternal and Child Health; Nobuhiko Okamoto, Department of Medical Genetics, Osaka Medical Center and Research Institute for Maternal and Child Health; Yoko Miyoshi, Noriyuki Namba, Keiichi Ozono, Department of Pediatrics, Osaka University Graduate School of Medicine; Yoshikazu Nishi, Department of Pediatrics, Hiroshima Red Cross Hospital. We also thank Julian Tang, Department of Education for Clinical Research, National Center for Child Health and Development, for reviewing and editing this manuscript.

\section{STATEMENT OF FINANCIAL SUPPORT}

All authors have no financial relationships relevant to this article to disclose.

Disclosure: All authors have no conflicts of interest to disclose.

\section{REFERENCES}

1. Noonan JA. Hypertelorism with Turner phenotype. A new syndrome with associated congenital heart disease. Am J Dis Child 1968;116:373-80.

2. Romano AA, Allanson JE, Dahlgren J, et al. Noonan syndrome: clinical features, diagnosis, and management guidelines. Pediatrics 2010;126:746-59.

3. Allanson JE, Hall JG, Hughes HE, Preus M, Witt RD. Noonan syndrome: the changing phenotype. Am J Med Genet 1985;21:507-14.

4. Nora JJ, Nora AH, Sinha AK, Spangler RD, Lubs HA. The Ullrich-Noonan syndrome (Turner phenotype). Am J Dis Child 1974;127:48-55.

5. Mendez HM, Opitz JM. Noonan syndrome: a review. Am J Med Genet 1985;21:493-506.

6. Noonan JA. Noonan syndrome and related disorders: alterations in growth and puberty. Rev Endocr Metab Disord 2006;7:251-5.

7. Roberts AE, Allanson JE, Tartaglia M, Gelb BD. Noonan syndrome. Lancet 2013;381:333-42.

8. Tartaglia M, Mehler EL, Goldberg R, et al. Mutations in PTPN11, encoding the protein tyrosine phosphatase SHP-2, cause Noonan syndrome. Nat Genet 2001;29:465-8.

9. Aoki Y, Niihori T, Banjo T, et al. Gain-of-function mutations in RIT1 cause Noonan syndrome, a RAS/MAPK pathway syndrome. Am J Hum Genet 2013;93:173-80.

10. Tartaglia M, Gelb BD. Disorders of dysregulated signal traffic through the RAS-MAPK pathway: phenotypic spectrum and molecular mechanisms. Ann NY Acad Sci 2010;1214:99-121.

11. Tartaglia M, Zampino G, Gelb BD. Noonan syndrome: clinical aspects and molecular pathogenesis. Mol Syndromol 2010;1:2-26.

12. van der Burgt I. Noonan syndrome. Orphanet J Rare Dis 2007;2:4.

13. van der Burgt I, Berends E, Lommen E, van Beersum S, Hamel B, Mariman E. Clinical and molecular studies in a large Dutch family with Noonan syndrome. Am J Med Genet 1994;53:187-91.

14. Nagai T, Matsuo N, Kayanuma Y, et al. Standard growth curves for Japanese patients with Prader-Willi syndrome. Am J Med Genet 2000;95:130-4.
15. Styles ME, Cole TJ, Dennis J, Preece MA. New cross sectional stature, weight, and head circumference references for Down's syndrome in the UK and Republic of Ireland. Arch Dis Child 2002;87:104-8.

16. Martin ND, Smith WR, Cole TJ, Preece MA. New height, weight and head circumference charts for British children with Williams syndrome. Arch Dis Child 2007;92:598-601.

17. Isojima T, Yokoya S, Ito J, Horikawa R, Tanaka T. New reference growth charts for Japanese girls with Turner syndrome. Pediatr Int 2009;51: 709-14.

18. Isojima T, Yokoya S, Ito J, Naiki Y, Horikawa R, Tanaka T. Proposal of new auxological standards for Japanese girls with turner syndrome. Clin Pediatr Endocrinol 2010;19:69-82.

19. Witt DR, Keena BA, Hall JG, Allanson JE. Growth curves for height in Noonan syndrome. Clin Genet 1986;30:150-3.

20. Malaquias AC, Brasil AS, Pereira AC, et al. Growth standards of patients with Noonan and Noonan-like syndromes with mutations in the RAS/ MAPK pathway. Am J Med Genet A 2012;158A:2700-6.

21. Ranke MB, Heidemann P, Knupfer C, Enders H, Schmaltz AA, Bierich JR. Noonan syndrome: growth and clinical manifestations in 144 cases. Eur J Pediatr 1988;148:220-7.

22. Ogata T, Matsuo N, Tamai S, Osano M, Tango T. Target height and target range for the Japanese (in Japanese). Jpn J Pediatr 1990;94:1535-40.

23. Wright EM, Royston P. A comparison of statistical methods for age-related reference intervals. J R Statist Soc A 1997;160:47-69.

24. Cole TJ. Fitting smoothed centile curves to reference data. J R Statist Soc A 1988;151:385-418.

25. Prendiville TW, Gauvreau K, Tworog-Dube E, et al. Cardiovascular disease in Noonan syndrome. Arch Dis Child 2014;99:629-34.

26. Otten BJ, Noordam C. Growth in Noonan syndrome. Horm Res 2009;72:Suppl 2:31-5.

27. Noonan JA, Raaijmakers R, Hall BD. Adult height in Noonan syndrome. Am J Med Genet A 2003;123A:68-71.

28. Binder G, Grathwol S, von Loeper K, et al. Health and quality of life in adults with Noonan syndrome. J Pediatr 2012;161:501-505.e1.

29. Tartaglia M, Kalidas K, Shaw A, et al. PTPN11 mutations in Noonan syndrome: molecular spectrum, genotype-phenotype correlation, and phenotypic heterogeneity. Am J Hum Genet 2002;70:1555-63.

30. Shaw AC, Kalidas K, Crosby AH, Jeffery S, Patton MA. The natural history of Noonan syndrome: a long-term follow-up study. Arch Dis Child 2007;92:128-32.

31. Zenker M, Buheitel G, Rauch R, et al. Genotype-phenotype correlations in Noonan syndrome. J Pediatr 2004;144:368-74.

32. Limal JM, Parfait B, Cabrol S, et al. Noonan syndrome: relationships between genotype, growth, and growth factors. J Clin Endocrinol Metab 2006;91:300-6.

33. Sarkozy A, Conti E, Seripa D, et al. Correlation between PTPN11 gene mutations and congenital heart defects in Noonan and LEOPARD syndromes. J Med Genet 2003;40:704-8.

34. Cole TJ, Green PJ. Smoothing reference centile curves: the LMS method and penalized likelihood. Stat Med 1992;11:1305-19.

35. Tanaka T, Yokoya S, Kato N, et al. Fundamental policies on evaluating auxological data for Japanese children (in Japanese). J Jpn Ass Hum Auxo 2011;17:84-99. 